\title{
Calls for routine collection of patient-reported outcome measures (PROMs) are getting louder
}

\author{
Nils Gutacker, PhD \\ Centre for Health Economics \\ University of York \\ Heslington \\ YO10 5DD \\ United Kingdom \\ email: nils.gutacker@york.ac.uk \\ (Corresponding author)
}

\author{
Andrew Street, PhD \\ Department of Health Policy \\ London School of Economics and Political Science \\ London \\ WC2A $2 A E$ \\ United Kingdom \\ email: a.street@lse.ac.uk
}

Most people use health services in the hope of improving their health or avoiding future deteriorations. It is remarkable, therefore, that although health systems collect large amounts of data on what they do (i.e. processes) and how much they do (activity) very little is known about whether it improves their patients' health (outcomes). This is not because data collection is unfeasible. Patient-reported outcome measures (PROMs), which are validated instruments to collect information from patients about their health problems and healthrelated quality of life (HRQoL), have existed for at least 40 years and are successfully employed in the context of clinical trials and health technology assessment. ${ }^{1}$ If it is possible to collect PROMs as part of trials, why not in routine care? And if it was to be implemented in routine care, would it be worth the cost?

There have long been advocates for the routine collection of measures of patient outcomes that go beyond survival statistics, including Thomas Percival (1740-1804) and Florence Nightingale (1820-1910) to name but a few. An early form of routine (albeit clinician-reported) outcome assessment followed from the 1845 UK Lunacy Act, which required physicians working in mental and acute hospitals to rate each patient discharged from their care as either dead, recovered, relieved, or unrelieved. But this requirement was met with resistance, notably from the medical profession, which was concerned that the data could not be compared meaningfully across patients. ${ }^{2}$ Moreover, following the introduction of the UK National Health Service (NHS) in 1948 policymakers focused on meeting the ever-increasing demand for care and this form of routine outcome measurement was abandoned shortly after. 
There now seems to be increased willingness to attempt another push at establishing routine collection of outcome measures, as reported by patients themselves. In 2017, health ministers from a variety of OECD countries signalled their support for the collection of data about patient-reported experiences and outcomes to augment existing country-level statistics. ${ }^{3}$ In some places this data collection is already happening. For instance, some clinical registries, most notably in the domain of hip and knee arthroplasty, have begun to routinely record their patients' HRQoL before and after surgery. ${ }^{4}$ However, there has not yet been widespread adoption across health systems and clinical settings, partly because data collection is costly and the expense has to be justified by the value of information the data yield.

The costs and benefits of routine PROM data collection have not been established definitively ${ }^{5}$ but some evidence is emerging from the national PROM programme in the English NHS, one of the most ambitious and extensive examples of routine data collection in recent times. Since 2009, all patients undergoing four types of NHS-funded surgery in English hospitals have been invited to report their HRQoL before and after treatment using generic and disease-specific instruments. Crucially, all providers of NHS-funded care must invite all their eligible patients to complete the questionnaires, thereby reducing the potential for selective recruitment of patients. By 2018 , over one million patients ( $60 \%$ of those eligible) had provided pre- and postoperative data at an estimated cost of $£ 6.5 \mathrm{~m}$, which proves that routine collection is feasible but not costless. ${ }^{6}$ These before-and-after data have been used in a variety of ways, for example to compare and financially incentivise the performance of hospitals 7 , to stimulate quality improvement work ${ }^{8}$, or to inform patients' choices of where to have surgery ${ }^{9}$.

Stakeholders' interest in PROMs is often squarely on the change in $H R Q O L$ that results from treatment, particularly where variation relates to the provider of care. The pre-treatment (i.e. baseline) HRQoL assessments on their own have received considerably less attention, even though they contain valuable information about the health status of those receiving care. Such data are typically missing from administrative data records even though the information is vital to our understanding of socio-economic inequalities in access to care or geographic variation in clinical admission thresholds. And the data could be used for more practical purposes, such as to involve patients in shared decision-making or as a screening device by physicians to identify patients with different care needs.

One example of such screening is the paper by Sutherland et al. in this issue ${ }^{10}$. The authors collected patient-reported measures of general health, depression and pain from over one thousand patients scheduled for general surgery in Vancouver, Canada. Perhaps not unexpectedly, they find that pain and depression correlate with the length of subsequent inpatient stay, even after controlling for patients' observable characteristics and the DiagnosisRelated Group (DRG) to which they have been assigned. More worryingly though, some patients reported symptoms consistent with severe depression even though this was not noted in their administrative records. The authors argue that some of the excess cost of moderate or severe depression, which are estimated to be $\$ 1,667$ and $\$ 3,270$, respectively, might be avoided if these patients were successfully identified through routine PROM collection, with their surgery not being initiated until their depression had been addressed. Clearly, more work is needed to establish how best to manage surgery for people with 
complex health problems. Nevertheless, the study exemplifies the potential value of pretreatment HRQoL data in the wider context of risk prediction and decision-making.

In the past those calling for routine collection of PROMs data were few and far between. Even now there remains a long way to go before PROMs data are collected in a routine fashion in all care settings and health systems, but there are promising signs that progress is being made. Problems with routine PROM collection exist, such as interpretability of the data, reporting biases, and how PROMs complement other types of information, but they are not insurmountable. New ideas for potential uses of these data are emerging rapidly but rigorous evaluations of most applications are still missing and the apparent use of these data cannot yet be equated with tangible health benefits to patients. As more data become available and are put to use, this will strengthen the evidence-base about the positive impacts that PROMs have on policy and practice. If these positive impacts outweigh the costs of data collection, calls to roll-out data collection will become louder. After all, without such information, it is difficult to establish the impact that health services have on improving our health.

\section{References}

1. Fitzpatrick R. Patient-reported outcome measures and performance measurement. In: Peter C Smith EM, Irene Papanicolas, Sheila Leatherman, ed. Performance Measurement for Health System Improvement. Cambridge: Cambridge University Press 2009:63-86.

2. Street $A$. The resurrection of hospital mortality statistics in England. Journal of Health Services Research \& Policy 2002;7(2):104-10.

3. OECD Health Ministers. The next generation of health reforms: ministerial statement, 2017.

4. Rolfson O, Eresian Chenok K, Bohm E, et al. Patient-reported outcome measures in arthroplasty registries. Acta Orthopaedica 2016;87(sup1):3-8.

5. Greenhalgh J, Dalkin S, Gibbons E, et al. How do aggregated patient-reported outcome measures data stimulate health care improvement? A realist synthesis. Journal of Health Services Research \& Policy 2018;23(1):57-65.

6. NHS England. Patient Reported Outcome Measures (PROMs) 2018 [Available from: https://digital.nhs.uk/data-and-information/data-tools-and-services/dataservices/patient-reported-outcome-measures-proms.

7. Gutacker N, Bojke C, Daidone S, et al. Hospital Variation in Patient-Reported Outcomes at the Level of EQ-5D Dimensions: Evidence from England. Medical Decision Making 2013;33(6):804-18.

8. Basser MR. Benefits case study - 'Patient Reported Outcome Measures (PROMs)' outputs: NHS Digital, 2015.

9. Gutacker N, Siciliani L, Moscelli G, et al. Choice of hospital: Which type of quality matters? Journal of Health Economics 2016;50:230-46.

10. Sutherland J, Liu G, Crump T, et al. Relationship between preoperative patient-reported outcomes and hospital length of stay: a prospective cohort study of general surgery patients in Vancouver, Canada. Journal of Health Services Research \& Policy; forthcoming. 